\title{
Chemical Treatment of Membranes of a Polymer Blend: Mechanism of the Reaction of Hypochlorite with Poly(vinyl pyrrolidone)
}

\author{
1. M. WIENK, E. E. B. MEULEMAN, Z. BORNEMAN, Th. VAN DEN BOOMGAARD,* and C. A. SMOLDERS \\ University of Twente, P. O. Box 217, 7500 AE Enschede, The Netherlands
}

\begin{abstract}
SYNOPSIS
Sodium hypochlorite solutions are used to treat membranes prepared from a polymeric blend containing poly(vinyl pyrrolidone) (PVP) to increase their water permeability. Sodium hypochlorite affects the membrane material in such a way that PVP is selectively removed from the membrane matrix. The mechanism of the reaction between hypochlorite and PVP is investigated by several chemical analysis techniques of the reaction products. Strong indications are found that the reaction involves chain scission of PVP according to a radical mechanism. (C) 1995 John Wiley \& Sons, Inc.
\end{abstract}

Keywords: polymer membranes • poly(vinyl pyrrolidone) • hypochlorite

\section{INTRODUCTION}

Membranes obtained by phase inversion of a polymer solution containing substantial amounts of the macromolecular additive poly(vinyl pyrrolidone) (PVP) usually show a low permeability for water. ${ }^{1}$ The low water flux is ascribed to swelling of the hydrophilic PVP present at the interface of the pore walls of the membrane resulting in decreased pore sizes. In Figure 1 and Table $I$ it is shown that upon treating ultrafiltration membranes of poly (ether sulfone) (PES) and PVP with a sodium hypochlorite solution membranes with a higher flux and a decreased PVP content are obtained. Roesink et al. ${ }^{2,3}$ found that treatment of microfiltration membranes of polyetherimide (PEI) and PVP with a sodium hypochlorite solution also increased the water flux by decreasing the PVP content of the membranes. The positive influence of PVP on the membrane properties-namely, giving it a hydrophilic character-is hardly affected by this treatment.

The reaction of PVP and sodium hypochlorite at pH 11.5 was studied earlier by Roesink. ${ }^{2}$ Two possible explanations were given for the selective removal of PVP from the membrane:

\footnotetext{
* To whom all correspondence should be addressed.
} Journal of Polymer Science: Part A: Polymer Chemistry, Vol. 33, 49-54 (1995) (C) 1995 John Wiley \& Sons, Inc. CCC 0887-624X/95/010049-06
1. Reaction of PVP with sodium hypochlorite causes chain scission of the polymer. This was confirmed by viscosity measurements. Since the molecular weight of PVP is decreased it can be washed out of the membrane matrix more easily.

2. Reaction of PVP with sodium hypochlorite causes ring opening of the pyrrolidone ring of the PVP molecule. The reaction is considered as an oxidation of PVP in alkaline solution. According to Roesink, ${ }^{2}$ the change of the chemical structure of PVP diminishes the interaction of this polymer with PEI and removal of PVP by washing the membrane is facilitated.

The possibility of ring opening of the pyrrolidone group in alkaline solution is reported by other authors ${ }^{4,5}$ also, but Roesink could not find direct evidence for this mechanism. The experiments that will be presented here will give more clarity on the mechanism of the reaction of PVP with sodium hypochlorite.

\section{THEORY}

Sodium hypochlorite $(\mathrm{NaOCl})$ is often used as a bleaching chemical for textile fibers. The effect of 


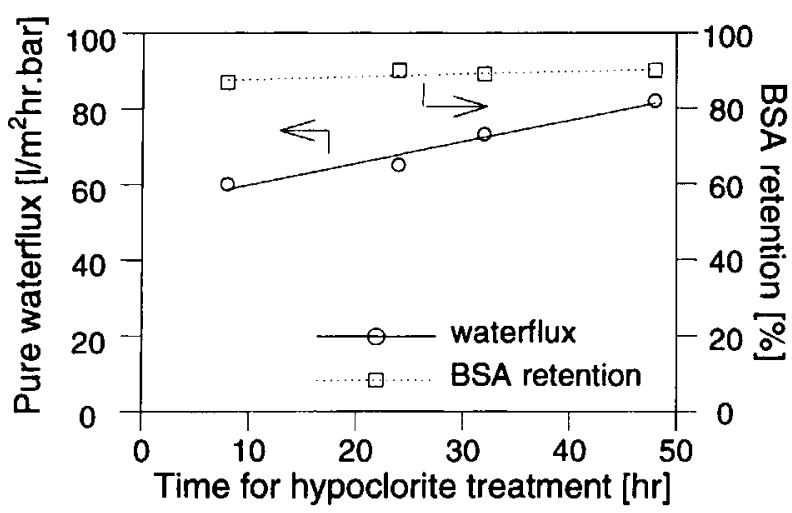

Figure 1. Pure water flux and retention for a $1 \mathrm{~g} / 1 \mathrm{BSA}$ solution of PES/PVP ultrafiltration membranes treated with a $4000 \mathrm{ppm}$ hypochlorite solution for different times.

hypochlorite on cellulose fibers is therefore studied extensively. ${ }^{6}$ Hypochlorite is a nonspecific oxidizing agent and its activity strongly depends on the $\mathrm{pH}$ of the reaction medium. Hypochlorite can attack ether bonds and hydroxyl groups and it is capable of cleaving $\mathrm{C}-\mathrm{H}$ bonds as well. According to Lewin, ${ }^{6}$ the oxidizing reactions are fastest at $\mathrm{pH}$ between 2 and 7.5 when the chlorite is in the protonated form $(\mathrm{HOCl})$. The general oxidizing reaction can be given by the equation:

$$
\mathrm{HOCl}+\text { substrate } \rightarrow \text { substrate }{ }^{\circ} \mathrm{O}+\mathrm{HCl}
$$

This reaction will cause lowering of the $\mathrm{pH}$ of the solution. According to Holst, ${ }^{7}$ free radicals play an active role in the oxidation with hypochlorite. A free radical chain is set-up as follows:

$$
\begin{aligned}
\mathrm{HOCl}+\mathrm{OCl}^{-} & \rightarrow{ }^{\circ} \mathrm{ClO}+\mathrm{Cl}^{-}+{ }^{\circ} \mathrm{OH} \\
\mathrm{OH}+\mathrm{OCl}^{-} & \rightarrow \mathrm{ClO}+\mathrm{OH}^{-} \\
\mathrm{ClO}+\mathrm{OCl}^{-}+\mathrm{OH}^{-} & \rightarrow 2 \mathrm{Cl}^{-}+\mathrm{O}_{2}+\cdot \mathrm{OH}
\end{aligned}
$$

Table I. PVP Content of the Membranes ${ }^{\mathrm{a}}$

\begin{tabular}{cc}
$\begin{array}{c}\text { Hypochlorite Treatment } \\
\text { (h) }\end{array}$ & $\begin{array}{c}\text { PVP/PVP + PES } \\
\text { (wt \%) }\end{array}$ \\
\hline & \\
8 & 4.6 \\
24 & 2.3 \\
32 & 1.3 \\
48 & 1.2 \\
\hline
\end{tabular}

\footnotetext{
a The PVP content was determined using micro element anal-
}

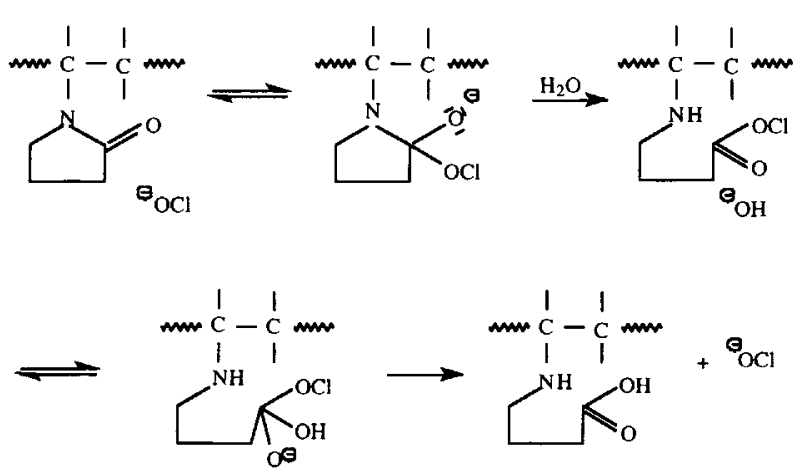

Scheme 1. Reaction mechanism for the reaction of PVP with hypochlorite in alkaline solution; ring-opening of PVP.

According to this scheme the radical ${ }^{\circ} \mathrm{OH}$ will react with the chain. However, in alkaline solutions so dium hypochlorite in water can be given as:

$$
\mathrm{HOCl}+\mathrm{OH}^{-} \rightarrow \mathrm{OCl}^{-}+\mathrm{H}_{2} \mathrm{O}
$$

and $\mathrm{OCl}^{-}$is the oxidant.

As said in the Introduction, the reaction of PVP in alkaline media can take place by opening of the pyrrolidone ring to form $\gamma$-amino acid units. The mechanism of this reaction is shown in Scheme 1. It is analogous to the reaction mechanism for the hydrolysis of lactams in aqueous solutions of potassium hydroxide. ${ }^{4}$

If radicals are involved in the reaction between hypochlorite and PVP, a hydrogen atom can be abstracted from the tertiary carbon atom, via disproportionation of the radical chain scission can occur. Another possibility is oxidative degradation by which an aldehyde (or carbonyl group) is formed. The reactions described here (Scheme 2) are taken from Anderson et al. ${ }^{8}$ These authors presented the reactions as side reactions that might take place during crosslinking of PVP using persulfate. In the presence of strong acid or alkali opening of the pyrrolidone ring may also take place by a radical reaction.

It is assumed that the reaction of PVP and hypochlorite takes place according to either one or both of the mechanisms mentioned above. Since the reactions strongly depend on $\mathrm{pH}$, for the experiments that are presented here three reaction media are used at $\mathrm{pH} 3.9,6.9$, and 11.5. Treatment of membranes containing PVP always takes place at $\mathrm{pH}$ 11.5. In these alkaline solutions opening of the pyrrolidone ring is expected to be the main reaction. ${ }^{2,4,5}$ Evidence for the reaction mechanism is based on chemical 


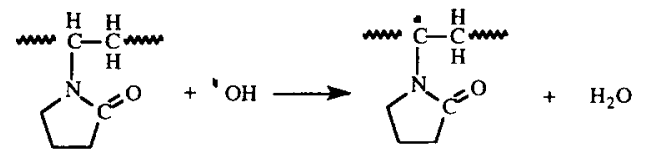

a)

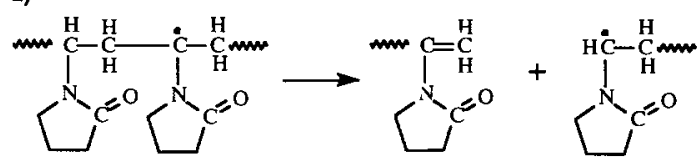

b)

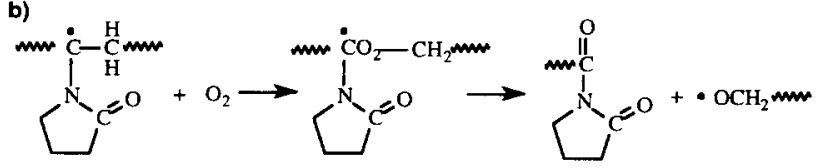

Scheme 2. Chain scission of PVP via radical reactions: (a) disproportionation and (b) oxidative degradation.

and structural analysis of the reaction products. To prevent any effect of residual hypochlorite this is removed from the reaction mixture by dialysis.

\section{EXPERIMENTAL}

\section{Materials}

Poly(vinyl pyrrolidone) (PVP) was purchased from Janssen Chimica, K90. Poly(ether sulfone) (PES) from ICI (Victrex 5200P) was used. Sodium hypochlorite ( $\mathrm{NaOCl}$ ) was purchased from Chemproha (technical quality, 15\% activity).

\section{Reaction}

The reaction solution contained $3000 \mathrm{ppm} \mathrm{NaOCl}$. The activity of the chlorite was verified by iodometric titration. The $\mathrm{pH}$ of this solution is 11.5. Two solutions were made of lower $\mathrm{pH}$ (6.9 and 3.9) by adding hydrochloric acid. Five grams of PVP was dissolved in $100 \mathrm{~mL}$ hypochlorite solution. The reaction took place for $48 \mathrm{~h}$ at room temperature. Then the low molecular weight components (salts) were removed from the reaction medium by dialysis (dialysis membrane, Tamson $256 \mathrm{k} 06$; pore size, $25 \AA$ ). The chlorine concentration of the dialysate was tested by adding silver nitrate. After 9 days of dialysis no chlorine could be detected anymore. After freeze drying the solution, a white powder was obtained which was used for analysis. A reference sample was obtained by dissolving PVP in water without hypochlorite, followed by dialysis of the solution, and freeze drying of the unreacted polymer.

\section{Analysis of the Reaction Products}

To obtain ${ }^{13} \mathrm{C}-\mathrm{NMR}$ (Bruker AC250) spectra PVP was dissolved in chloroform $\left(\mathrm{CDCl}_{3}\right)$. Infrared spectra (IR) were obtained by using an IR apparatus of Nicolet 5SXC.

The number of acidic or alkaline groups of the reaction product can be determined by potentiometric titration in nonaqueous solutions.

Microelement analysis (MEA) was used to determine the $\mathrm{C} / \mathrm{N}$ weight ratio of the reaction product. For this method, the powder is completely oxidized in a graphite furnace oven at $1800^{\circ} \mathrm{C}$. The amount of elemental carbon and nitrogen can be determined by gas chromatography of the reaction products.

GPC measurements were performed using $\mu$ Styragel columns $\left(10^{5}+10^{4}+10^{3} \AA\right)$ and a Guard column (500 $\AA$ ). Molecular weight was determined by LALLS (Chromatix KMX-6) and by a refractive index detector (Differential Refractometer, Waters 411).

\section{RESULTS}

The reaction of PVP with hypochlorite was performed at three different $\mathrm{pH}$ values of the reaction medium. After 2 days of reaction, the $\mathrm{pH}$ was again measured. For the three solutions the $\mathrm{pH}$ decreased from 11.5 to 11.4 , from 6.9 to 1.8 , and from 3.9 to 1.4 , respectively. Since $\mathrm{pH}$ decreases due to the formation of $\mathrm{HCl}$ during oxidation, this indicates that oxidation has taken place at $\mathrm{pH} 3.9$ and 6.9. Any oxidation that might have taken place at $\mathrm{pH} 11.5$ can not be detected by change in $\mathrm{pH}$ because an excess of alkali is not detected by $\mathrm{pH}$.

In the ${ }^{13} \mathrm{C}-\mathrm{NMR}$ spectra no differences could be found between the reacted and unreacted PVP. If opening of the pyrrolidone ring had occurred, a peak of the carboxylic group would appear at a shift of $182.5 \mathrm{ppm}$. Chain scission would give two peaks of the alkene group at a shift of 94 and $130 \mathrm{ppm}$. These peaks were not found in the spectra.

By infrared analysis a small peak was found (in the region $1760-1780 \mathrm{~cm}^{-1}$ ) that could be ascribed to an ester group or an acid group. The first group could be the result of a crosslinking bond after oxidative degradation of a radical. If the peak represents an acid group it is an indication that ring opening had taken place. This peak was found only in the samples reacted at $\mathrm{pH} 6.9$ and 3.9. The sample of pH 11.5 showed exactly the same spectrum as the reference. If alkene groups would be present in large 
Table II. Potentiometric Titration of Carboxylic Groups Present in PVP after Reaction of the Polymer with Hypochlorite at Three $\mathrm{pH}$ Values as well as the Unreacted Polymer ${ }^{a}$

\begin{tabular}{lcccc}
\hline & $\begin{array}{c}\text { Carboxylic Groups } \\
\text { Acidic Form } \\
\text { PVP Sample }\end{array}$ & $\begin{array}{c}\text { Totkaliv/g) } \\
(\mu \text { equiv/g) }\end{array}$ & $\begin{array}{c}\text { Total } \\
(\mu \text { equiv/g) }\end{array}$ & $\begin{array}{c}\text { Ring-Opening } \\
(\%)\end{array}$ \\
\hline Reference & $<5$ & $10.0 \pm 0.3$ & 15.0 & 0.17 \\
pH 11.5 & $63.2 \pm 0.4$ & $46.9 \pm 2.6$ & 110.1 & 1.22 \\
pH 6.9 & $<5$ & $102.6 \pm 8.4$ & 107.6 & 1.19 \\
pH 3.3 & $<5$ & $79.2 \pm 11.7$ & 84.2 & 0.93 \\
\hline
\end{tabular}

a The carboxylic groups are ascribed to openirig of the pyrrolidone rings. In the last column the number of opened rings is expressed as percentage of the total number of pyrrolidone rings.

b The number of pyrrolidone rings is $9 \mathrm{mmol} / \mathrm{g}\left[=1 / M_{w}(\mathrm{PVP}\right.$ monomer $\left.)\right]$.

enough quantities this should result in an additional peak in the IR spectra (in the region $1600-1700$ $\mathrm{cm}^{-1}$ ) which was not observed.

Potentiometric titration of the reaction products was performed to verify the presence of carboxylic groups. Both acidic and alkaline groups were found with an acidic strength comparable to propionic acid and sodium propionate, respectively. This indicates that the acidic and alkaline groups result from opening of the pyrrolidone unit of PVP. The percentage of the pyrrolidone rings that were opened during reaction of PVP with hypochlorite can be calculated from the number of acidic and alkaline groups. For all three $\mathrm{pH}$ values only $1 \%$ ring opening occurred (data are listed in Table II). At $\mathrm{pH} 11.5$ the carboxyl group was present mainly in the alkaline form.

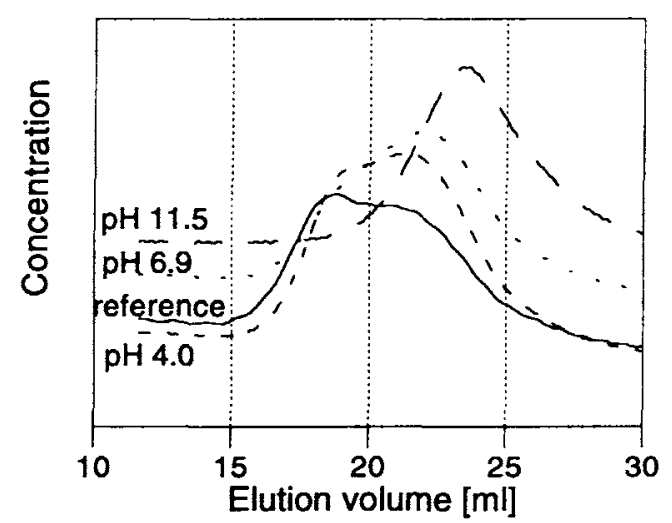

Figure 2. The results of GPC measurements. Distribution curves of the molecular weight of PVP after reaction of the polymer with hypochlorite at three $\mathrm{pH}$ values as well as the unreacted polymer are shown. The distribution curve is plotted as concentration versus the elution volume. The elution volume is inversely proportional to the molecular weight of the polymer.
The ratio of nitrogen and carbon atoms of the samples is determined using MEA. The $\mathrm{C} / \mathrm{N}$ ratio was found to be 5.2 for the reacted as well as unreacted PVP. The ratio $\mathrm{C} / \mathrm{N}$ calculated from the molecular structure of PVP is 5.14. With MEA no information on addition of oxygen can be found since it can not be detected with this technique.

The molecular weight of PVP before and after reaction was determined using GPC. The molecular weight distributions of the three reaction products and the reference are shown in Figure 2. The weight average molecular weight and the number-average molecular weight of the products are listed in Table III. From GPC data, it can be concluded that the molecular weight of PVP decreases upon reaction with hypochlorite. The chain scission is most effective at $\mathrm{pH} 11.5$ and the reaction also diminishes the polydispersity of the polymer.

The GPC technique is applicable only if no structural changes of the molecule have occurred. If, during the reaction, charged groups are introduced, the dimension of the molecule might change because of repulsive or attractive forces. To verify the effect of charged groups on the molecular weight distribution

Table III. Weight-average Molecular Weight and Number-average Molecular Weights of PVP after Reaction of the Polymer with Hypochlorite at Three pH Values as well as the Unreacted Polymer as Determined using GPC

\begin{tabular}{lccc}
\hline $\begin{array}{c}\text { PVP } \\
\text { Sample }\end{array}$ & $\begin{array}{c}M_{w} \times 10^{3} \\
(\mathrm{~g} / \mathrm{mol})\end{array}$ & $\begin{array}{c}M_{n} \times 10^{3} \\
(\mathrm{~g} / \mathrm{mol})\end{array}$ & $M_{w} / M_{n}$ \\
\hline Reference & 997 & 242 & 4.12 \\
pH 11.5 & 170 & 141 & 1.21 \\
pH 6.9 & 612 & 156 & 3.92 \\
pH 3.3 & 763 & 182 & 4.19 \\
\hline
\end{tabular}




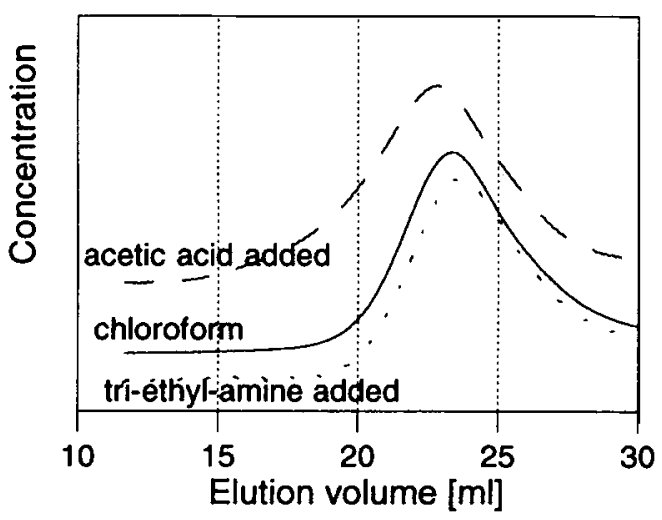

Figure 3. Checking whether the eventual presence of charged groups in PVP affect the molecular weight distribution curves as measured using GPC.

as found by GPC two extra measurements were performed. Either acetic acid or tri-ethyl amine was added to the solution to diminish (if relevant) the number of charged groups present in PVP. For all samples the three curves obtained were almost equal. For $\mathrm{pH} 11.5$, the results of these measurements are shown in Figure 3.

Any effect of the dialysis step was investigated by measuring three reference samples: PVP as obtained from the supplier, PVP dissolved in water and dried by freeze-drying, and PVP dissolved in water, dialyzed, and dried. For all the analysis techniques used (NMR, IR, MEA, GPC) no difference between the three samples could be found.

The membrane-forming polymer PES is not ex-

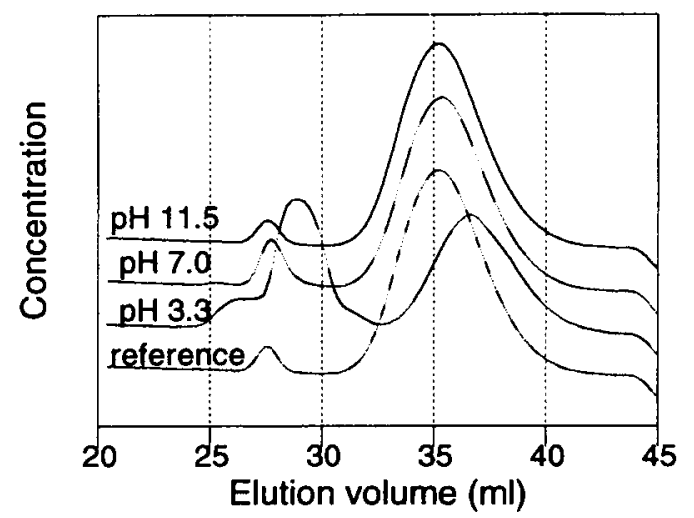

Figure 4. Distribution curves of the molecular weight of PES after reaction of the polymer with hypochlorite at three $\mathrm{pH}$ values as well as the unreacted polymer as determined using GPC. The distribution curve is plotted as concentration versus the elution volume. The elution volume is inversely proportional to the molecular weight of the polymer.
Table IV. Weight-Average Molecular Weight and Number-Average Molecular Weights of PES after Reaction of the Polymer with Hypochlorite at Three $\mathrm{pH}$ Values as well as the Unreacted Polymer as Determined using GPC

\begin{tabular}{lcc}
\hline $\begin{array}{c}\text { PES } \\
\text { Sample }\end{array}$ & $\begin{array}{c}M_{w} \times 10^{3} \\
(\mathrm{~g} / \mathrm{mol})\end{array}$ & $\begin{array}{c}M_{n} \times 10^{3} \\
(\mathrm{~g} / \mathrm{mol})\end{array}$ \\
\hline Reference & 56.6 & 28.0 \\
pH 11.5 & 58.9 & 27.5 \\
pH 6.9 & 68.0 & 34.3 \\
pH 3.3 & 45.6 & 32.0 \\
\hline
\end{tabular}

pected to react with hypochlorite. Chain scission of PES upon hypochlorite treatment is unfavorable because this would largely decrease the mechanical strength of the membranes. To be sure that the molecular weight of PES is not affected by hypochlorite, GPC measurements have been performed. Since PES is not soluble in water it was dispersed in the hypochlorite solution and the reaction took place under severe stirring of the solution. The molecular weight distribution of PES after reaction and dialysis was determined using GPC (see Fig. 4 and Table IV). At pH 6.9 and 11.5, the molecular weight distribution of the reacted polymer was equal to that of the unreacted polymer. However, at $\mathrm{pH} 3.9$, a large high molecular weight fraction was found (probably caused by crosslinking) and also the low molecular weight fraction was higher.

\section{DISCUSSION}

The ${ }^{13} \mathrm{C}$-NMR spectra do not show structural changes of PVP after reaction of the polymer with hypochlorite. The extra peak found by IR can be ascribed to the carboxyl group indicating that a ringopening reaction had occurred. However, at $\mathrm{pH} 11.5$, the peak was not found whereas ring opening was expected to take place most frequent in alkaline solution. Titration of acid and alkaline groups of PVP indicate that only $1 \%$ of the pyrrolidone groups had been opened during reaction. This percentage was the same for all three $\mathrm{pH}$ values.

Structural changes of PVP are only small and any charged groups present did not influence the GPC data. Therefore, the data found using GPC can be interpreted as caused by a decrease of the molecular weight due to reaction with hypochlorite. The decrease in molecular weight resulting from chain scission of the polymer is strongest at $\mathrm{pH}$ 11.5. 
Based on the chain scission mechanism of PVP the molecular weight after reaction at $\mathrm{pH} 11.5$ is 170,000 (see Fig. 2 and Table III). The number of end groups of the polymers present is less than 2 for every 1000 monomer groups. The number of carboxylic groups found by titration of the reaction product is 1 for every 100 monomer groups, which is apparently too low to be detected with ${ }^{13} \mathrm{C}-\mathrm{NMR}$. It is therefore reasonable that end groups resulting from chain scission can not be detected by ${ }^{13} \mathrm{C}-\mathrm{NMR}$ or IR.

\section{CONCLUSIONS}

Upon reaction of PVP with sodium hypochlorite structural changes of PVP could not be detected using ${ }^{13} \mathrm{C}-\mathrm{NMR}$ and IR. Titration measurements indicate that $1 \%$ of the pyrrolidone rings are opened. Thus, only minor structural changes have occurred. On this basis, it can be concluded from GPC data, which do show a definite shift in elution volume, that the molecular weight of PVP decreases due to reaction of the polymer with hypochlorite. The decrease in molecular weight is highest at $\mathrm{pH} 11.5$. The decrease in molecular weight of PVP is caused by chain scission according to a radical mechanism.

The membrane forming polymer (PES) is fairly resistant to treatment with a hypochlorite solution, especially at $\mathrm{pH} 11.5$, which is the $\mathrm{pH}$ for the aftertreatment of the membranes.

The authors acknowledge J. F. J. Engbersen for the enlightening discussions on this subject.

\section{REFERENCES AND NOTES}

1. I. M. Wienk, Ph. D. Thesis, Twente University, Enschede, 1993.

2. H. D. W. Roesink, Ph. D. Thesis Twente University, Enschede, 1989.

3. H. D. W. Roesink, D. M. Koenhen, M. H. V. Mulder, and C. A. Smolders, U.S. Pat. 4,798,847.

4. M. I. Vinnik and Y. V. Moiseyev, Tetrahedron, 19, 1441 (1963).

5. P. Molyneux, Water-Soluble Synthetic Polymers: Properties and Behavior, Vol. I, CRC Press, Boca Raton, FL, 1982, p. 146.

6. M. Lewin, Bleaching of cellulosic and synthetic fabrics, in Handbook of Fiber Science and Technology, Vol. 2: Chemical Processing of Fibers and FabricsFunctional Finishes, M. Lewin and S. B. Sello, eds., Dekker, New York, 1985, p. 91.

7. G. Holst, Chem. Rev., 54, 169 (1954).

8. C. C. Anderson, F. Rodriguez, D. A. Thurston, J. Appl. Polym. Sci., 23, 2453 (1979).

Received October 18, 1993

Accepted July 5, 1994 